\title{
Application of Six Sigma DMAIC Methodology to Reduce Medication Errors in a Major Trauma Care Centre in India
}

\author{
Ankitha George ${ }^{1, *}$, Aida Mary Joseph ${ }^{1}$, Sreenath Kolencherry ${ }^{1}$, Vishnu Vettekkaram Kodath ${ }^{1}$, Kaliyan \\ Menaka $^{2}$, Britto Duraisingh ${ }^{3}$, Sheik Haja Sherief ${ }^{4}$, Thangavel Shivakumar ${ }^{5}$
}

${ }^{1}$ Pharm D Interns, Department of Pharmacy Practice, Nandha College of Pharmacy, Erode, Tamil Nadu, INDIA. ${ }^{2}$ Assistant Professor, Department of Pharmacy Practice, Nandha College of Pharmacy, Erode, Tamil Nadu, INDIA. ${ }^{3}$ Clinical Pharmacist, Ganga Medical Centre Hospital, Coimbatore, Tamil Nadu, INDIA.

${ }^{4} \mathrm{HOD}$, Department of Pharmacy Practice, Nandha College of Pharmacy, Erode, Tamil Nadu, INDIA.

${ }^{5}$ Principal, Nandha College of Pharmacy, Erode, Tamil Nadu, INDIA.

\begin{abstract}
Introduction: Medication error is a continuing global phenomenon. It is an important public health problem that poses a serious threat to patient safety. This study was carried out to analyze the effect of Six Sigma methodology in reducing medication errors in a tertiary care hospital. Method: A Prospective study on application of Six Sigma DMAIC methodology to reduce medication errors were conducted during a period of February 2017 to July 2017 at Ganga Medical College Hospital, Coimbatore, Tamil Nadu. Results: Out of 1050 cases, the number of prescribing, transcribing, dispensing, administering and monitoring errors were found to be $62,19,6,47$ and 14 in the measure phase which reduced to $12,10,2,7$ and 4 in the improve phase. Conclusion: Implementation of DMAIC methodology showed a marked reduction in medication errors, correspondingly the sigma value improved from 3.56, 3.93, 4.33, 3.63 and 4.1 to $4.15,4.16,4.66,4.33$ and 4.33 . Regular auditing and proper follow up can result in sustaining the improvement in the medication use process.
\end{abstract}

Key words: Six Sigma, Medication errors, Adverse drug events, Error outcome category.

\section{INTRODUCTION}

Medication error is any preventable event that may cause or lead to inappropriate medication use or patient harm while the medication is in the control of the health care professional, patient or consumer. ${ }^{1}$ Such events may be related to professional practice, healthcare product, procedures and systems, including prescribing; order communication; product labelling; packaging and nomenclature; compounding; dispensing; distribution; administration; education; monitoring and use. ${ }^{2}$

Medication errors and preventable adverse drug events have a major number of public health implications ranging from increased length of stay at the hospital, detrimental effects on quality of life and even death. The medication errors are one among the most common health threatening mistakes that affect patient care. Such mistakes are considered as a global problem which leads to increased mortality rates, length of hospital stay and related costs. Approximately 1 to $10 \%$ of medication errors are associated with patient harm worldwide..$^{3-5}$ Almost every patient receiving healthcare receives medicines as part of their care which is unavoidable. Literally, millions of doses per year are administered to patients in an average hospital and billions of doses are self-administered by the patient in the outpatient setting. The elderly are found to have the highest rate of death from medication errors. ${ }^{6}$ Several factors present in the geriatric population contribute to this increased risk including polypharmacy,
DOI: 10.5530/ijopp.11.4.38

Address for correspondence: Dr. Ankitha George, Doctor of Pharmacy Intern, Department of Pharmacy Practice, Nandha College of Pharmacy, Erode, Tamil Nadu, INDIA.

Phone no: +917907320996 Email Id: ankithamareena694@ gmail.com

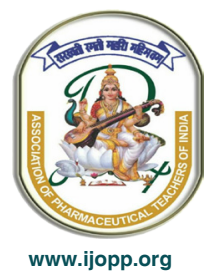


increased drug sensitivity, drug-disease interactions and unnecessary drug utilization. ${ }^{7}$

Preventing errors saves money and saves lives. Through the application of quality improvement methodologies and thereby safety measures in health care processes, we can significantly reduce medication errors. The American Society of Health System Pharmacists (ASHP) definition of medication errors includes prescribing, dispensing, medication administration and patient compliance errors which are to be reduced. The National Coordinating Committee on Medication Error Reporting and Prevention (NCCMERP) in the United States is an interdisciplinary healthcare group which consist of representatives of fourteen healthcare organizations. Their purpose is to promote the reporting, understanding and eradication of medication errors and focus on the way to protect patient safety through the coordinated efforts of associations and agencies.

Six Sigma is considered to be one of the best quality improvement methodology which presently finds its application in healthcare. ${ }^{8}$ The joint commission for accreditation of healthcare organization require hospitals to implement projects to improve their patient care process. ${ }^{9}$ Basically Six Sigma measures 3.4 defects per million of opportunities (DPMO) and it operate on the concept of DMAIC methodology. ${ }^{10}$

\section{Objectives}

The objective of the current study is to reduce the number of prescribing, transcribing, dispensing, administering and monitoring errors using Six Sigma DMAIC methodology and to measure the error outcome category.

\section{METHODOLOGY}

\section{Study design}

This study was a prospective study using Six Sigma DMAIC methodology to reduce medication errors in a major trauma care centre in India. The study was conducted at Department of Orthopaedics and Plastic Surgery, Ganga Hospital, Coimbatore for a period of six months with the sample size of 1050 cases.

\section{Inclusion and exclusion criteria}

All patients admitted in the hospital were included in the study. All mentally retarded patients, drug addicts and unconscious patients unable to respond to verbal questions were excluded from the study.

\section{Method of data collection}

A total of 1050 cases were collected during the study period from ICU, NICU and HDU. Patients who met the inclusion criteria were followed till the day of discharge. The study period was divided into five phases according to study methodology. The data was collected using well-structured data collection form which included patient demographics, medical and medication history, drugs prescribed, type of ME, error outcome category, factors causing ME, personnel causing $\mathrm{ME}$ and interventions done. The clinical progress notes of physician were used to evaluate the clinical outcome of the patient during follow up days. Appropriateness of medication usage was analysed from patient's case sheet regularly and all the discrepancies observed have been documented appropriately in the data collection form designed for the study.

\section{Data analysis}

The information collected regarding all the selected cases were recorded in a master chart and analyzed using Graph pad version 5.03. Chi square test was used to compare the results and $\mathrm{P}$ value of $<0.05$ was considered to be significant.

\section{DMAIC METHODOLOGY \\ Define Phase}

The define phase is to make clear understanding of scope and objective. Also, the purpose of the project and scope will be defined during the phase. One of the key major success factors of Six Sigma project is that, it starts with understanding of what service processes are critical to achieve the objectives. During the define phase, baseline data was obtained to identify the occurrence of medication errors in the hospital. The nature of the problem was determined to be that errors occurred more frequently in administration and prescription. A total of 100 cases were collected and the results are shown in the Table 1.

\section{Measure phase}

In the measure phase, a data collection sheet was prepared to obtain data and to identify what type of errors were occurring. The DPU, DPMO and Sigma values for the respective errors were also calculated. Measure performance phase focused on the distribution, collection and refinement of the different types of medication errors. Pareto analysis was done to identify which factor resulted in most number of errors. Prescribing errors mostly occurred due to wrong dose prescribed (Figure 1), 


\begin{tabular}{|ccc|}
\hline \multicolumn{3}{|c}{ Table 1: Baseline Data in the Define Phase. } \\
\hline Type of Errors & Number of Errors & Percentage \\
\hline Prescribing Errors & 25 & $45.45 \%$ \\
Transcribing Errors & 4 & $7.27 \%$ \\
Dispensing Errors & 1 & $1.82 \%$ \\
Administering Errors & 21 & $38.18 \%$ \\
Monitoring Errors & 4 & $7.27 \%$ \\
Total & 55 & $100 \%$ \\
\hline
\end{tabular}

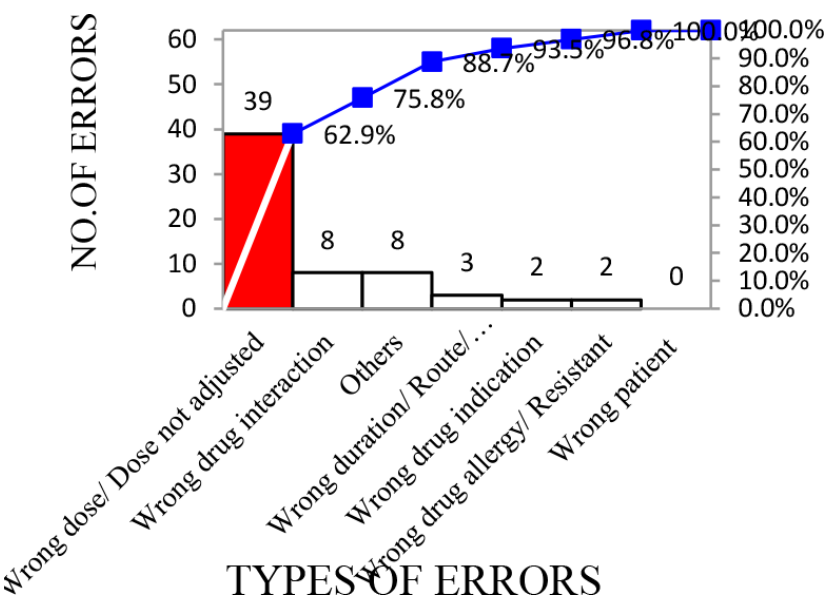

Figure 1: Measure phase prescribing errors
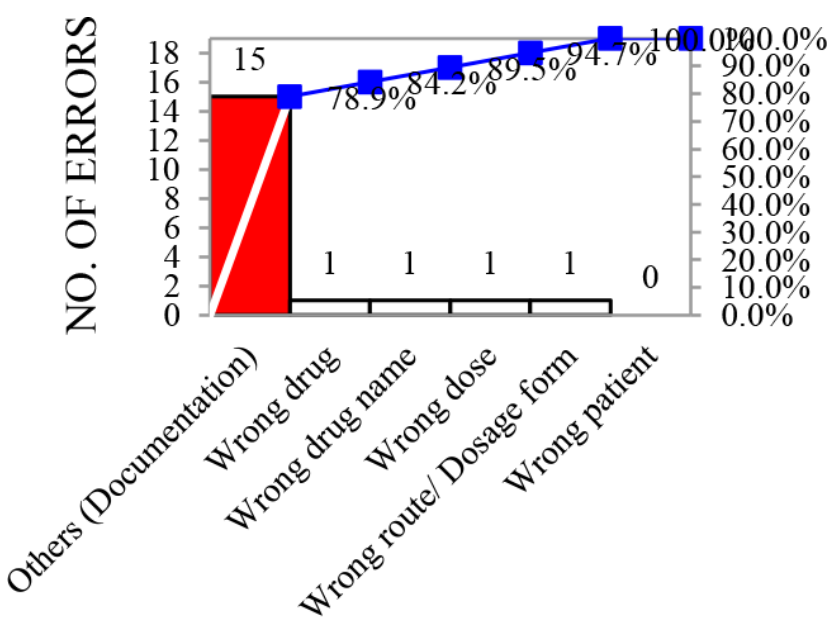

\section{TYPES OF ERRORS}

Figure 2: Measure phase transcribing errors

transcribing errors due to wrong documentation (Figure 2), dispensing errors due to wrong patient and wrong dose (Figure 3) administering errors due to wrong time (Figure 4) and monitoring errors due to results not acted upon (Figure 5) from the Pareto charts.

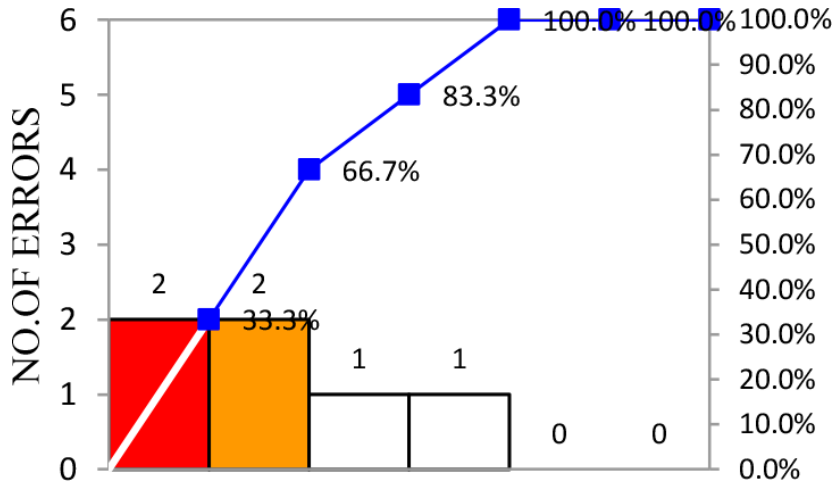

Wrong Wrong Wrong Wrong Wrong Others patient dose drug no.of dosage drugs form

\section{TYPES OR ERRORS}

Figure 3: Measure phase dispensing errors

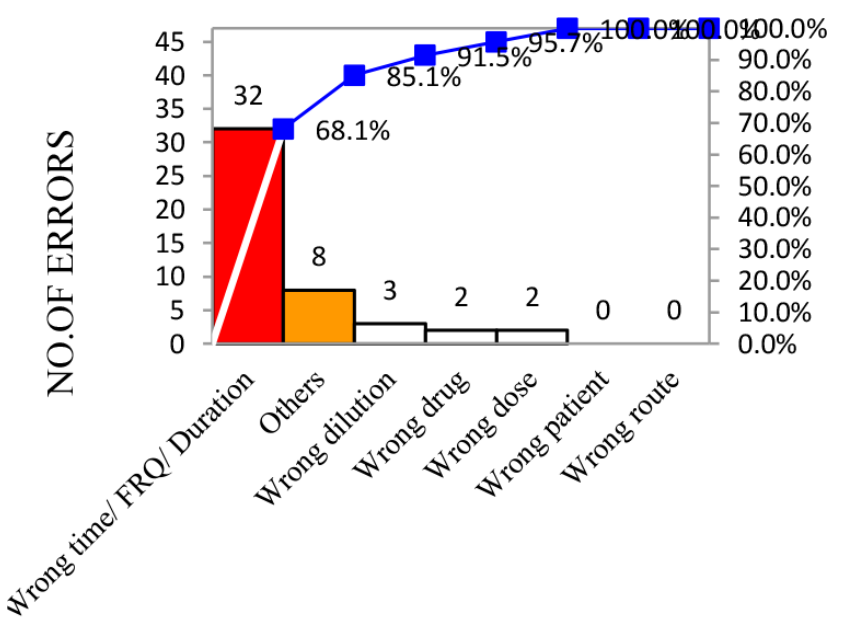

TYPES OF ERRORS

Figure 4: Measure phase administering errors

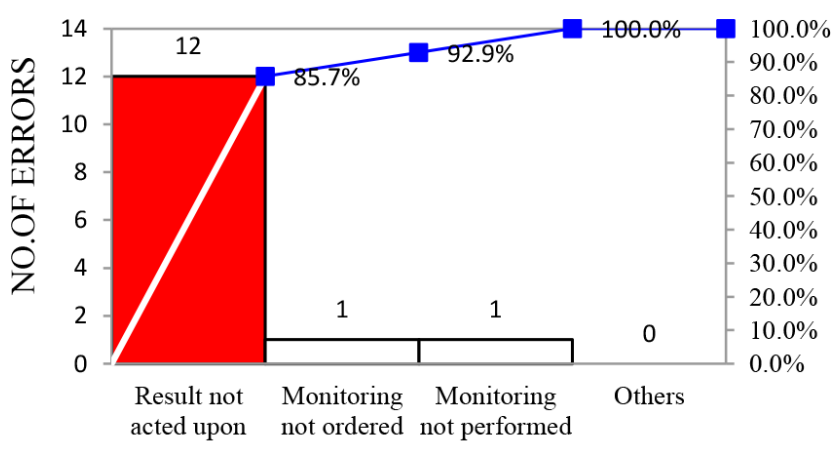

TYPES OF ERRORS

Figure 5: Measure phase monitoring errors

Indian Journal of Pharmacy Practice, Vol 11, Issue 4, Oct-Dec, 2018 


\section{Analyze phase}

The team discovered why defects are generated by detecting the key variables that are most likely to create process variation. It is of high importance to analyse the process in order to fix the source where high medication error occurs. In this Phase Fish bone analysis was used for the Root Cause Analysis.

\section{Improve phase}

The improve phase mainly involved brainstorming potential solutions with the research team for selecting the best risk reduction opportunities, testing and evaluating the implemented actions. The aim of improve phase was to identify the corrective methods that can be useful for the identified problems during analysis phase.

\section{Control phase}

During the control phase, a control chart was further created to find out if the error rates are sustained. The major aim of control phase is to sustain the improvements obtained during the process. Tools are put in place to ensure that under the corrected process the key variables remain within the maximum acceptable ranges over time.

\section{RESULTS AND DISCUSSION}

A total of 1050 cases were collected for the study purpose. In this study, DMAIC was applied in five phases such as Define, Measure, Analyze, Improve and Control.

In the define phase, a project charter was developed which included title, clinical need, goal and scope of the project. A control chart was prepared with 100 cases to evaluate the major types of errors that were prescribing, dispensing, transcribing, administering and monitoring. From the define phase, the prescribing errors were 25(45.45\%), transcribing errors were 4(7.27\%), dispensing errors were $1(1.82 \%)$, administering errors were $21(38.18 \%)$ and monitoring errors were $4(7.2 \%)$.

In the measure phase, a total of 62 prescribing errors were detected among which $2(3.23 \%)$ wrong drug indication, $2(3.23 \%)$ wrong drug allergy/resistant, $8(13 \%)$ wrong drug interaction, $39(63 \%)$ wrong dose/dose not adjusted, $3(4.83 \%)$ wrong duration/ route/frq, $8(13 \%)$ others were identified.

These sub classes of prescribing errors were plotted on a Pareto chart for further analysis. From the Pareto chart, wrong dose errors were highlighted to be focused on. This was similar to that of the study conducted by Chaital Nikil Shah in 2013. ${ }^{11}$ For route cause analysis, fishbone diagram was used and the factors for wrong dose errors were detected as lack of knowledge, inexperienced professional, not considering comorbid conditions and not calculating doses for paediatrics, geriatrics, hepatic and renal patients. In the improve phase, action plan to reduce the wrong dose errors was prepared. The corrective measures included conducting classes for physicians, stock taking, pharmacist audits; regular auditing of prescription to prevent wrong dose errors, ensuring effective communication; physicians can clarify any doubts regarding doses with clinical pharmacist, presenting interventions in the core committee meeting, notifying prescription error to the consultants on time to rectify the error occurred, sharing the clinical interventions to consultants through mail, conducting separate audits on the observed issues and submitting to consultants and getting suggestions from senior consultants to avoid observed errors. Followed by in the improve phase, 425 cases were collected to observe significant reduction in prescribing errors. The number of prescribing errors were found to be 12 with the rise in sigma value from 3.54 to 4.15 . In the control phase, 100 cases were collected and a control chart was prepared to observe the controlled number of errors.

\begin{tabular}{|c|c|c|c|c|c|c|}
\hline \multirow[t]{2}{*}{ TYPE OF ERRORS } & \multirow{2}{*}{$\begin{array}{l}\text { MEASURE } \\
\text { PHASE }\end{array}$} & \multirow{2}{*}{$\begin{array}{l}\text { IMPROVE } \\
\text { PHASE }\end{array}$} & \multirow{2}{*}{$\begin{array}{l}\text { MEASURE } \\
\text { PHASE \% }\end{array}$} & \multirow{2}{*}{$\begin{array}{l}\text { IMPROVE } \\
\text { PHASE\% }\end{array}$} & \multicolumn{2}{|c|}{ SIGMA LEVELS } \\
\hline & & & & & PHASE II & PHASE IV \\
\hline PRESCRIBING ERRORS & 62 & 12 & $14.59 \%$ & $2.82 \%$ & 3.54 & 4.15 \\
\hline TRANSCRIBING ERRORS & 19 & 10 & $4.47 \%$ & $2.35 \%$ & 3.93 & 4.16 \\
\hline DISPENSING ERRORS & 6 & 2 & $1.41 \%$ & $0.47 \%$ & 4.33 & 4.66 \\
\hline ADMINISTERING ERROR & 47 & 7 & $11.06 \%$ & $1.65 \%$ & 3.65 & 4.33 \\
\hline MONITORING ERRORS & 14 & 4 & $3.29 \%$ & $0.94 \%$ & 3.9 & 4.33 \\
\hline NO ERROR & 277 & 390 & $65.18 \%$ & $91.77 \%$ & - & - \\
\hline TOTAL & 425 & 425 & 100 & 100 & - & - \\
\hline$X^{2}$ VALUE & \multicolumn{6}{|c|}{21.155} \\
\hline P VALUE & \multicolumn{6}{|c|}{ 0.0008(Significant) } \\
\hline
\end{tabular}


The errors in transcribing were measured as 19 in the measure phase. The major sub type of transcribing error that was highlighted by Pareto analysis was wrong documentation error. Fish bone analysis was performed to detect the major causing factors as lack of communication, illegibility of prescriptions in drug chart, unfilled elements of prescription, poor knowledge on drugs, overworked nurses, unaware of importance of documentation, lack of interest, poor medication reconciliation practice. The corrective measures to reduce the number of transcribing errors were developed in the action plan which included, educating nurses regarding the importance of documentation, during peak hours more number of staffs posted to avoid errors, regular audits by pharmacist, doctors are asked to fill the drug chart themselves, doctors signature to be placed after each prescribed drugs, importance of medication reconciliation has been strengthened by education, major issues were informed to nursing superintendent to take necessary actions by coordinating with nursing educators, the issues observed were strengthened. A reduction in number of transcribing error was measured to 10 after the improve phase. The number of errors were found to be reduced to 10. The Sigma value increased from 3.93 to 4.16 .

The number of dispensing errors in the define phase was found to be 6 . In the measure phase 6 were detected. The subclasses of dispensing errors was found to be $2(33.33 \%)$ wrong patient, $1(16.67 \%)$ wrong drug, $2(33.33 \%)$ wrong dose and 1(16.67\%) wrong number of drugs from the Pareto analysis. From the fish bone analysis the root causes for dispensing errors were detected to be peak hour, patient demands which result in confusion, not checking stock, lack of space in pharmacy and aggressive patients. Action plan was generated which included measures such as more number of staffs posted during peak hours, double check before dispensing regular stock audits, patient turn-around time monitoring etc. Followed by the improve phase, 425 cases were collected to observe significant reduction in dispensing errors. The number of dispensing errors were found to be 2 with the rise in sigma value from 4.33 to 4.66 . This was similar to the study conducted by Ahmed Al Kuwaiti in 2016. ${ }^{12}$

The errors in administering were measured as 47 in the measure phase. The major sub type of administering error that was highlighted by Pareto analysis was wrong time error. Fishbone analysis was performed to detect the major causing factors as incomplete prescription, increased work load for nurses, lack of staffs, forgetful nurses and lack of knowledge. Corrective measures included preparation of dilution chart for drugs, provided adequate number of staffs, education and training

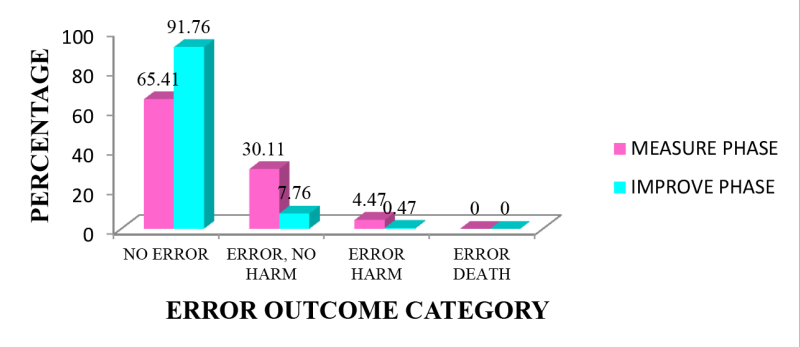

Figure 6: Error Outcome Category

provided, questionnaire prepared, exams conducted for nurses and regular pharmacist audits. The number of errors reduced to 7 and the sigma value increased from 3.65 to 4.33 .

A total of 14 monitoring errors were detected among which $1(7.14 \%)$ monitoring not ordered, 1 (7.14\%) monitoring not performed, $12(85.71 \%)$ result not acted upon. These sub classes of monitoring errors were plotted on a Pareto chart for further analysis. From the Pareto chart monitoring not performed was the subclass to be focused on. Fish bone diagram was used and the factors were identified as busy physicians, lack of interest, not aware of importance of monitoring and lack of communication. Corrective actions included classes conducted to educate physicians, nurses educated regarding effective communication, physicians asked to sign in laboratory reports and regular audits by pharmacist. In the end, the errors reduced to 4 and the sigma value raised from 3.9 in the improve phase to 4.33 in the measure phase.

The error outcome category consisted of no error, errorno harm, error-harm, error-death. The measure phase showed 278, 128, 19 and 0 respectively. It improved to 390, 33, 2 and 0 respectively in the improve phase which is depicted in the below diagram Figure 6.

\section{CONCLUSION}

In our study, implementation of Six Sigma DMAIC methodology showed a marked reduction in prescribing, transcribing, dispensing, administering and monitoring errors. Correspondingly the Sigma values has improved from 3.56, 3.93, 4.33, 3.63, 4.1 to 4.15, 4.16, 4.66, 4.33, 4.33 respectively. From the study it was concluded that the medication use process is complex and various strategies were utilized to safeguard against medication errors such as improving communication, the use of technology, education and development of policies for high-risk medications. Optimization of this process involved learning from past events-medication errors. In conclusion, it is apparent that the application of the 
Six Sigma error reduction methodology is extremely powerful in identifying, quantifying and controlling complex hospital systems.

\section{ACKNOWLEDGEMENT}

We express our heartfelt gratitude to our Professors of Department of Pharmacy Practice, Doctors, Clinical pharmacists of Ganga Hospital, Coimbatore, friends and also thank the patients who participated in our study.

\section{CONFLICT OF INTEREST}

The author declares no conflict of interest.

\section{ABBREVIATIONS}

DMAIC: Define Measure Analyze Improve Control; NCCMERP: The National Coordinating Committee on Medication Error Reporting and Prevention; ICU: Intensive Care Unit; NICU: Neonatal Intensive Care Unit; HDU: High Dependency Unit; DPU: Defects Per Unit; DPMO: Defects Per Million Opportunities.

\section{SUMMARY}

In the project, Six Sigma DMAIC Methodology was used successfully to reduce medication errors significantly. Corresponding improvement in the Sigma values were also ascertained.

\section{REFERENCES}

1. Parthasarathi G, Karin Nyfort Hansen, Nahata MC. A textbook of clinical pharmacy practice. Second edition. Hyderabad. Universities press publishing; 2004.

2. Parry K. Association For The Study Of Medical Education Report Of A Conference Held At The Royal College Of Physicians, London On 29 May 1987. Medical Education. 1987;21(6):512-20.

3. Zaal RJ, Doormaal JEV, Lenderink AW, Mol PG, Kosterink JG, Egberts TC, et al. Comparison of potential risk factors for medication errors with and without patient harm. Pharmacoepidemiologic Drug Safety. 2010;19(8):825-33.

4. Stavroudis TA, Shore AD, Morlock L, Hicks RW, Bundy D, Miller MR. NICU medication errors: identifying a risk profile for medication errors in the neonatal intensive care unit. J Perinatol. 2010;30(7):459-68.

5. Crespin DJ, Modi AV, Wei D, Williams CE, Greene SB, Pierson S, Hansen RA. Repeat medication errors in nursing homes. Contributing factors and their association with patient harm. American Journal of Geriatric Pharmacotherapy. 2010;8(3):258-70.

6. Phillips J, Beam S, Brinker A, Holquist C, Honig P, Lee LY, et al. Retrospective analysis of mortalities associated with medication errors. American Journal of Health System Pharmacist. 2001;58(19):1835-41.

7. Hanlon JT, Schmader KE, Ruby CM, Weinberger M. Suboptimal prescribing in older inpatients and outpatients. Journal of American Geriatric Society. 2001;49(2):200-9.

8. Cathy B. Application of Six Sigma to Reduce Medical Errors. ASQ's $55^{\text {th }}$ Annual Quality Congress. 2001;55:739-42

9. Allen TT, Tseng SH, Swanson K, McClay MA. Improving the hospital discharge process with Six Sigma methods. Quality Engineering. 2009;22(1):13-20.

10. Nandakumar PM, Anitha HS, Bhanu PS. Six Sigma Methodology Utilization in Medical Transcription-A DMAIC Process to Identify Six Sigma Projects. International Journal of Scientific Engineering and Technology. 2014;3(5):574-8.

11. Chaital NS, Nilay S. Clinical Research: Medication Errors and its Root Cause Analysis in Multispecialty Hospital. International Journal of Pharmacy Teaching and Practices. 2013;4(4):838-43.

12. Ahmed AK. Application of six sigma methodology to reduce medication errors in the outpatient pharmacy unit: a case study from the king Fahd university hospital, Saudi Arabia. International Journal for Quality Research. 2016;10(2):267-78. 